\title{
Rol de los lisados bacterianos en asma, evidencia y proyección con especial referencia a $\mathrm{OM}-85$
}

\author{
DANIEL ZENTENO A.*, JAVIERA DÍAZ S.** y ARNOLDO QUEZADA L.***
}

\section{Role of bacterial lysates in asthma, evidence and projection with special reference to $\mathrm{OM}-85$}

There are several bacterial lysates, being OM-85 (Broncho-Vaxom $\left.{ }^{\circledR}\right)$ the one with the greatest evidence regarding its immunoprotective role on respiratory infections in the pediatric population. Its mechanisms of action produce immunomodulatory effects that could potentially prevent asthma in early stages of life, act on the reduction of crisis and be a contribution to conventional asthma therapy. This article shows the main evidences in relation to these compounds, the current focus and future development, especially on OM-85.

Key words: Bacterial lysate, asthma, OM-85, child.

\section{Resumen}

Existen diversos lisados bacterianos, siendo OM-85 (Broncho-Vaxom ${ }^{\circledR}$ ) el que posee mayor evidencia en cuanto a su rol inmunoprotector sobre infecciones respiratorias en población pediátrica. Sus mecanismos de acción producen efectos inmunomoduladores que potencialmente podrían prevenir el asma en etapas precoces de la vida, actuar sobre la disminución de crisis y ser un aporte a la terapia convencional del asma. Este artículo expone las principales evidencias en relación con estos compuestos, con enfoque en la actualidad y el desarrollo futuro, en especial sobre OM-85.

Palabras clave: Lisados bacterianos, asma, OM-85, niño.

\section{Introducción}

Los inmunoestimulantes orales están formados por un grupo muy variado de compuestos farmacológicos, dentro de estos, los lisados bacterianos son los que poseen mayor respaldo científico en la actualidad; siendo su principal representante OM-85 (Broncho-Vaxom ${ }^{\circledR}$ ). Este compuesto incluye 21 cepas bacterianas y es considerado una estrategia para la prevención y coadyuvante del tratamiento de las infecciones recurrentes del tracto respiratorio en adultos y niños, con amplia evidencia que confirma su eficacia en la disminución de la frecuencia de estas patologías ${ }^{1,2}$. Del Río Navarro y cols. en un metaanálisis publicado en Cochrane confirman que los estudios que incluyeron OM-85 eran los que poseían mejor evidencia en disminuir la frecuencia de infecciones respiratorias en niños en comparación con otros inmunoestimulantes, mostrando un 36\% de reducción en el número total de infecciones respiratorias agudas en comparación con placebo ${ }^{2}$.

Entre sus mecanismos de acción demostrados están los efectos antivirales mediados por citoquinas y la regulación de las subpoblaciones celulares que participan en la respuesta inflamatoria alérgica.

Con estos antecedentes es esperable plantear si pudiese existir un rol de este lisado bacteriano en la prevención de los orígenes del asma, en la mo-

* Pediatra Broncopulmonar, Servicio de Pediatría, Hospital Guillermo Grant Benavente, Concepción. Profesor Asistente, Departamento de Pediatría, Universidad de Concepción. Concepción, Chile.

** Interna medicina, Universidad de Concepción. Concepción, Chile.

*** Pediatra Broncopulmonar, Profesor Titular, Departamento de Pediatría y Cirugía Infantil, Campus Sur, Facultad de Medicina, Universidad de Chile. Santiago, Chile. 
dificación de su evolución natural, la reducción de crisis obstructivas y un potencial efecto favorable en el control de esta enfermedad, adicional a las terapias convencionales actuales.

El presente artículo entrega información según evidencias recientes y en desarrollo sobre asma y lisados bacterianos, con especial referencia a OM-85.

\section{Asma y rol de los lisados bacterianos}

En Chile, la prevalencia de asma es de aproximadamente un $15 \%$ de la población pediátrica, siendo una patología de consulta frecuente tanto para el médico general como para el especialista ${ }^{3}$.

Su tratamiento ha variado a través del tiempo; en los años 70s, se buscó revertir el broncoespasmo mediante el empleo de $\beta 2$ agonistas, logrando disminuir las muertes por crisis de asma. Posteriormente, se introdujeron los corticoides inhalados para manejar la inflamación, luego se incluyeron progresivamente otras estrategias para intentar influir sobre la remodelación bronquial y lograr un mejor control de síntomas, lo cual ha permitido lograr resultados beneficiosos evidentes en el control de esta patología, reducción de exacerbaciones y mejorar la calidad de vida de estos pacientes.

Actualmente, varios investigadores han orientado sus esfuerzos en la prevención del asma. La Academia Europea de Alergia e Inmunología planteó las potenciales estrategias de prevención de esta patología, incluyendo el uso de inmunomoduladores, como los lisados bacterianos, debido a su esperable potencialidad ${ }^{4}$.

\section{Lisados bacterianos}

Los lisados bacterianos corresponden a partículas fraccionadas mecánicamente o células liofilizadas químicamente degradadas, derivadas de bacterias patógenas responsables de infecciones del tracto respiratorio.

Desde la década de los 70 se han desarrollado diferentes productos comerciales dentro de la categoría de lisados bacterianos, destacando en población pediátrica: OM-85, PMBL (Ismigen), ribomunyl y LW 50020 (Luivac), los que incluyen extractos de microorganismos como Streptococcus pneumoniae, Haemophilus influenzae y Klebsiella pneumoniae ${ }^{5,6}$ (Tabla 1). Lisados bacterianos utilizados para la prevención de infecciones respiratorias en niños.

Administrados por vía oral, son absorbidos en el tracto digestivo llegando a la superficie del tejido linfoide donde son procesados y presentados a las células dendríticas, entonces se generan linfocitos $\mathrm{T}$ específicos que modulan a los linfocitos $\mathrm{B}$, mediante citoquinas, TGF- $\beta$ y miembros de la familia del factor de necrosis tumoral, para producir inmunoglobulina A ( $\operatorname{IgA}$ ). OM-85 interactúa con las células dendríticas a través de receptores tipo Toll (TLR) induciendo su maduración y la posterior migración como células presentadoras de antígenos profesionales. La secreción citoquímica que se genera promueve una respuesta inflamatoria donde se libera factor activador de células B, ligando inductor de proliferación, IL-6 y TGF- $\beta$ y aumenta la producción de IL-10 e interferón, citoquinas reconocidas como las más relevantes en la defensa contra las infecciones virales ${ }^{7}$.

La inmunomodulación consiste en un mejor

Tabla 1. Composición de lisados bacterianos utilizados para la prevención de infecciones respiratorias en niños

\begin{tabular}{|lcccc|}
\hline & OM-85 & PMBL & LW 50020 & Ribomunyl \\
\hline Streptococcus pneumoniae & Sí & Sí & Sí & Sí \\
Streptococcus pyogenes & Sí & Sí & Sí & Sí \\
Streptococcus viridans & Sí & Sí & - & - \\
Haemophilus infuenzae & Sí & Sí & Sí & Sí \\
Klebsiella pneumoniae & Sí & Sí & Sí & Sí \\
Klebsiella ozaenae & Sí & Sí & - & - \\
Moraxella catarrhalis & Sí & Sí & Sí & - \\
Staphylococcus aureus & Sí & Sí & Sí & - \\
Otros & & \multicolumn{3}{c}{ Streptococcus mitis } \\
\hline
\end{tabular}

Nombre comercial de compuestos: OM-85: Broncho-vaxom, PMBL: Ismigen, LW 50020: Luivac. 
balance de Th1 y Th2 mediante la reducción de las células T helper $2 \mathrm{CD} 4+$, junto con citoquinas inflamatorias (IL-4,5,6,13). Proceso asistido por las células $\mathrm{T}$ reguladoras que bloquean la producción de citoquinas. Esto se complementa con la inducción de citoquinas pro-T helper $1 \mathrm{y}$ el aumento de IL-10 en condiciones pro inflamatorias ${ }^{8}$ (Figura 1). Este conjunto de acciones favorece la disminución de las infecciones virales, causantes de las sibilancias y desencadenantes del inicio y evolución del asma. A su vez, en base a este mecanismo, se podría lograr un efecto sobre la atopía y el control de las infecciones ${ }^{9}$.

Recientemente, Liu y cols. utilizaron modelos de ratones asmáticos para tratarlos con OM-85 vía oral y analizar la infiltración de células inflamatorias; eosinófilos y neutrófilos. Tomaron muestras de tejido pulmonar y niveles de citoquinas de tipo Th1/Th2 en secreción de lavado broncoalveolar. Finalmente, comparado con el grupo control, se observó una reducción significativa del grosor de pared de la vía aérea, la estenosis luminal y la formación de tapones de moco, junto con una disminución significativa de la cantidad de eosinófilos en las muestras de lavado broncoalveolar. Con estos datos es posible atribuir un efecto positivo en los organismos afectados por asma ${ }^{10}$.

\section{Infecciones respiratorias, lisados bacterianos y asma}

Distintas cohortes en el mundo han descrito los fenotipos de asma considerando su relación a la edad de diagnóstico, gatillantes, comorbilidades y severidad; destacando aquellos individuos que presentan síntomas en una etapa precoz de la vida; muchos de ellos en forma transitoria y asociada a infecciones respiratorias virales ${ }^{11}$. En este grupo es donde varios investigadores han propuesto un rol en la prevención temprana de asma, mediante el uso de lisados bacterianos y su consecuente reducción de infecciones respiratorias.

En cuanto a la incidencia de asma, se ha demostrado a lo largo de los años que existe una relación altamente significativa con la infección grave por virus respiratorio sincicial y aún más con infección por rinovirus, la que se incrementa en casos de coinfección por estos ${ }^{12,13}$. Yin y cols. en un metaanálisis publicado en 2018, confirma lo demostrado en Cochrane por Del Río Navarro y cols. sobre la disminución de las infecciones respiratorias con el uso de OM-85, lo que ayudaría potencialmente a prevenir el desarrollo de asma en algunos sujetos. Realizando una búsqueda bibliográfica en PubMed, Embase, Cochrane

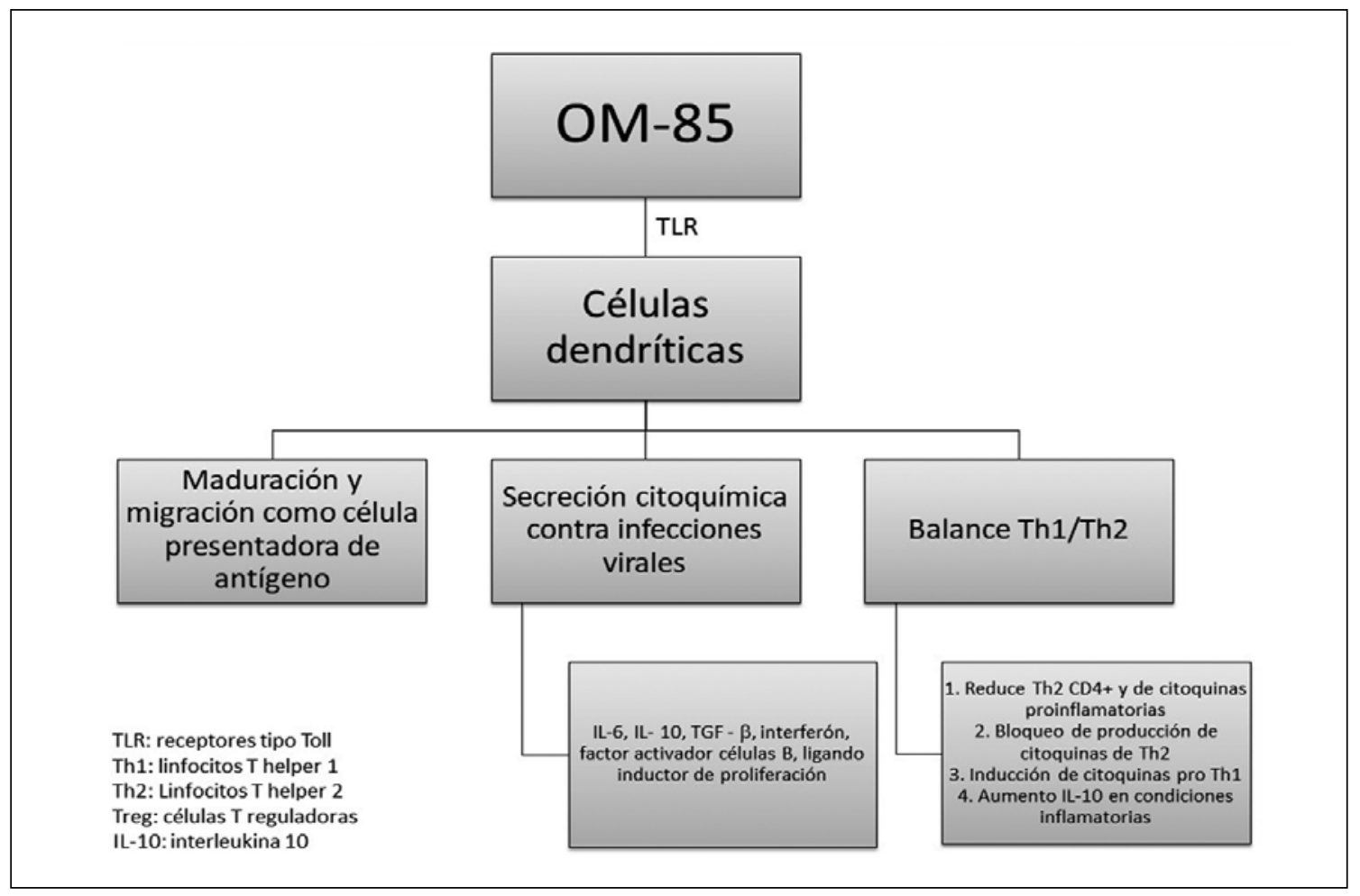

Figura 1. Inmunomodulación por OM-85. 
y las bases de datos CBM, CNKI y VIP, con un total de 4.851 pacientes pediátricos, lograron evidenciar en el grupo tratado, una reducción en el uso de antibióticos 4 días, tos 5 días y de la duración de episodios de sibilancias 3 días ${ }^{2,14}$. Adicionalmente sus resultados mostraron un aumento de los niveles de células T e inmunoglobulinas A, $\mathrm{G}$ y $\mathrm{M}$ tras el uso del lisado bacteriano ${ }^{14}$.

En relación con la forma y período de administración de OM-85, Chen et al, en el año 2007 utilizaron este inmunomodulador en esquema habitual de 10 días al mes por 3 meses en 50 pacientes con episodio de sibilancias atribuibles a infección respiratoria alta que necesitaron hospitalización, en los cuales 9 meses después se mostró una disminución significativa de los episodios en comparación al grupo que sólo fue tratado con budesonida $(25 \%$ versus $63 \%$; $<<0,05)$ teniendo como punto de corte 3 o más episodios de sibilancias durante el período de seguimiento ${ }^{15}$.

Liao y Zhang, en 2014 incorporaron OM-85 en 62 niños de 1 a 12 años con diagnóstico de asma, separándolos en un grupo control y un grupo que incluía OM-85 dentro de su tratamiento. Los investigadores midieron los niveles de Inmunoglobulinas A, G, M, urea y alaninaaminostransferasa al inicio, a los 6 y 12 meses. Tras un año de seguimiento constataron un aumento de los péptidos estimulantes de células $\mathrm{T}$, de $\operatorname{IgG}, \operatorname{Ig} \mathrm{A}$ y disminución de número de infecciones respiratorias, asociado a reacciones adversas leves como constipación y dolor abdominal en un pequeño número de pacientes ${ }^{16}$. Conclusiones similares se encontraron en el año 2015 integrando OM85 en el tratamiento de niños de 5 a 15 años con diagnóstico de asma, sumándose el hallazgo de un aumento de las células natural killer tras su empleo, información aportada por Lu y cols. en investigaciones realizadas en Shangai, China ${ }^{17}$. En el año 2010, el grupo de trabajo de Razi y cols. realizó un estudio doble ciego, randomizado con grupos paralelos donde se agregó OM-85 10 días al mes por 3 meses en niños de 1 a 6 años con sibilancias inducidas por virus respiratorios, de un total de 75 pacientes 35 recibieron inmunoestimulante versus 40 con placebo, con un seguimiento de 9 meses post administración del lisado, resultando en una disminución del número de crisis de hasta un $38 \%$, efecto que se mantenía e incluso aumentaba durante el período de observación. Adicionalmente demostraron una disminución en la duración de los días de crisis en los niños tratados ${ }^{18}$.

El efecto de otros lisados bacterianos ha sido evaluado especialmente en la prevención de infecciones respiratorias, sin embargo, en forma muy discreta en relación a asma ${ }^{19,20}$. Existen reportes en reducidos grupos de asmáticos que sugieren que el empleo de ribomunyl aumenta los niveles de linfocitos T, IL2 y reduce IL 1 y 6 , por lo tanto, posee un potencial efecto beneficioso en este grupo de pacientes ${ }^{20,21}$.

Recientemente, se dieron a conocer los resultados del estudio EOLIA (Efficacy Of mechanical bacterial Lysate In Allergic children), aleatorio, doble ciego y controlado con placebo, quienes evaluaron el efecto de lisados bacterianos (PMBL - Ismigen ${ }^{\circledR}$ ) en 152 pacientes asmáticos alérgicos, de 6 a 16 años; empleando el esquema de administración convencional, 1 dosis por 10 días durante 3 meses y realizando un seguimiento durante 9 meses más. En los casos tratados, el número de exacerbaciones fue $63 \%$ menor al tercer mes y $42 \%$ menor al final del período de estudio, resultados significativos en relación a gatillantes infecciosos. Adicionalmente, se destaca que el número de días con exacerbación fue un 33\% más bajo, los tiempos de aparición de la primera, segunda y tercera crisis se prolongaron un $30 \%$, $55 \%$ y $74 \%$ respectivamente ${ }^{22}$. Estos resultados orientan a considerar la indicación de lisados bacterianos en la terapia convencional de asma ${ }^{18,22}$.

Con respecto a otras patologías asociadas a asma, se ha reportado una reducción de la dermatitis atópica con el uso de OM-85. Destacamos los resultados de Lau y cols. quienes administraron este lisado bacteriano por 6 meses en promedio a lactantes con antecedente de un progenitor atópico y posterior seguimiento por 3 años, encontrando una menor incidencia de dermatitis atópica en comparación con el grupo placebo, situación significativa en hijos de padres atópicos $(11 \% \text { versus } 32 \%)^{23}$.

\section{Investigaciones futuras}

Actualmente, se están realizando interesantes investigaciones en diferentes partes del mundo en relación a lisados bacterianos, especialmente OM-85 y sus efectos sobre episodios de sibilancias o asma. En Australia, se ejecuta el estudio OMPAC (Protection against acute respiratory infections during early childhood using OM-85), que incluye 60 lactantes menores de 9 meses, con antecedentes familiares de asma o atopia, donde se administran 2 ciclos consecutivos del lisado; sus resultados estarán disponibles a finales de 2019. En EE.UU se ejecuta el ensayo clínico ORBEX (The oral bacterial extract study), estudio multicéntrico, controlado con placebo, que busca determinar el efecto de OM-85 en aumentar el 
Tabla 2. Estudios en curso sobre OM-85 en la prevención y tratamiento de asma

\begin{tabular}{|c|c|c|c|}
\hline Estudio & Diseño & Principales criterios de valoración & Duración \\
\hline $\begin{array}{l}\text { OMPAC } \\
\text { Australia }\end{array}$ & $\begin{array}{l}\text { - } 60 \text { lactantes (3-9 meses) } \\
\text { - Riesgo de asma/atopia (hermanos) } \\
\text { - OM-85 o placebo: } 3,5 \mathrm{mg} \text { ( } 10 \text { dosis/5 } \\
\text { meses consecutivos), } 2 \text { ciclos en las } \\
2 \text { primeras temporadas de invierno }\end{array}$ & $\begin{array}{l}\text { - Prevención primaria de la IVRS } \\
\text { durante los dos primeros años } \\
\text { - Desarrollo de asma persistente en } \\
\text { niños de alto riesgo } \\
\text { - Microbiota nasal, sensibilización } \\
\text { alergénica, análisis transcriptómico }\end{array}$ & $\begin{array}{l}2 \text { años con se- } \\
\text { guimiento de } \\
1 \text { año } \\
\text { Final: } 2019\end{array}$ \\
\hline $\begin{array}{l}\text { ORBEX } \\
\text { EE. UU. }\end{array}$ & $\begin{array}{l}\text { - } 1.076 \text { lactantes (6-18 meses) } \\
\text { - Alto riesgo de asma } \\
\text { - OM-85 o placebo: } 3,5 \mathrm{mg} \text { ( } 10 \text { días/ } \\
\text { mes durante } 2 \text { años consecutivos) }\end{array}$ & $\begin{array}{l}\text { Prevención de las infecciones respi- } \\
\text { ratorias de tracto superior } \\
\text { - ADN, suero, muestra de heces para } \\
\text { futuros marcadores virales, bacteria- } \\
\text { nos y genéticos }\end{array}$ & $\begin{array}{l}2 \text { años, segui- } \\
\text { miento de } 1 \text { año } \\
\text { Final: } 2021\end{array}$ \\
\hline $\begin{array}{l}\text { OMPeR } \\
\text { Italia }\end{array}$ & $\begin{array}{l}\text { - } 288 \text { niños (1-5 años) } \\
\text { - IVR recurrentes ( } \geq 6 / \text { año) } \\
\text { - Factores de riesgo especiales: inmu- } \\
\text { nodeficiencia leve, atopia o sibilan- } \\
\text { cias recurrentes } \\
\text { - OM-85 o placebo: } 3,5 \mathrm{mg}(10 / \text { dosis } \\
\text { durante } 3 \text { y } 6 \text { meses) }\end{array}$ & $\begin{array}{l}\text { Prevención de las infecciones respi- } \\
\text { ratorias de tracto superior } \\
\text { - Criterios secundarios: Duración de } \\
\text { IVRS, gravedad, número promedio, } \\
\text { amigdalitis bacteriana, otitis aguda, } \\
\text { días escolares perdidos, terapia y } \\
\text { ciclos antibióticos }\end{array}$ & $\begin{array}{l}6 \text { meses } \\
\text { Final: } 2018\end{array}$ \\
\hline $\begin{array}{l}\text { BREATE } \\
\text { (Países Bajos) }\end{array}$ & $\begin{array}{l}\text { - } 120 \text { pacientes ( } 12-40 \text { años) } \\
\text { - Asma no controlada } \\
\text { OM- } 85 \text { o placebo: } 7 \text { mg (10 días por } \\
\text { mes) durante } 2 \text { temporadas consecu- } \\
\text { tivas) }\end{array}$ & $\begin{array}{l}\text { - Reducción de las exacerbaciones de } \\
\text { asma } \\
\text { Microbioma nasofaríngeo/fecal y } \\
\text { marcadores inflamatorios en suero/ } \\
\text { esputo }\end{array}$ & $\begin{array}{l}18 \text { meses, segui- } \\
\text { miento de } 1 \text { año } \\
\text { Final: } 2021\end{array}$ \\
\hline
\end{tabular}

OMPAC: Protection against acute respiratory infections during early childhood using OM-85. ORBEX: The oral bacterial extract study. OMPeR: Efficacy and safety of OM-85 in children with recurrent respiratory tract infections. BREATHE: Infection prevention and immune modulation by bacterial lysates: gaining insight into the mechanism of an old therapy in patients with asthma. IVRs: infecciones de vía respiratoria superior.

tiempo de aparición del primer episodio de sibilancias por infección respiratoria en niños con eczema o familiar directo con asma; finalizará en 2021, luego de 2 años de tratamiento consecutivo y 1 año de seguimiento posterior. Italia por su parte desarrolla el ensayo OMPeR (Efficacy and safety of OM-85 in children with recurrent respiratory tract infections), que busca determinar el rol en prevención de infecciones de tracto de respiratorio en niños de 1 a 5 años, monitorizando además la duración y gravedad de la enfermedad, la necesidad de antibióticos y consecuencias; se espera den a conocer sus resultados próximamente. En Países Bajos, el estudio BREATHE (Infection prevention and immune modulation by bacterial lysates: gaining insight into the mechanism of an old therapy in patients with asthma) incluye adolescentes y adultos con edades entre 12 y 40 años con asma no controlada, quienes recibirán de forma aleatoria OM-85 por 2 inviernos consecutivos, realizando posteriormente 1 año se seguimiento, para evaluar el efecto en las exacerbaciones de asma $^{8}$ (Tabla 2).

En resumen, se encuentra disponible una amplia y variada literatura con relación a los li- sados bacterianos y próximamente se obtendrán los resultados de estudios en curso que incluyen distintos países y pacientes de diferentes edades que ayudarán a sustentar el rol de los lisados bacterianos en asma (Tabla 2). Actualmente, se conoce que los lisados bacterianos y principalmente OM-85, previenen las infecciones respiratorias agudas $y$, considerando esto como un desencadenante para el desarrollo de asma, podría formar parte de la prevención de la enfermedad, incidir en el curso natural de esta e incluso ser parte del tratamiento convencional de la patología para lograr una menor sintomatología y menor número de crisis.

\section{Bibliografía}

1.- ZENTENO D, QUEZADA A. Inmunoestimulantes orales en enfermedades respiratorias infantiles. Rev Ped al día 2007; 25: 38-42.

2.- DEL RÍO B, ESPINOSA F, FLENADY V, SIENRAMONGE J. Cochrane Review: Immunostimulants for preventing respiratory tract infection in children. Cochrane database of systematic reviews 2006; 18 (4).

3.- FORNO E, GOGNA M, CEPEDAA, YÁÑEZ A, SOLÉ 
D, COOPER P, et al. Asthma in Latin America. Thorax 2015; 70 (9): 898-905.

4.- EDWARDS MR, WALTON RP, JACKSON DJ, FELESZKO W, SKEVAKI C, JARTTI T, et al. The potential of anti-infectives and immunomodulators as therapies for asthma and asthma exacerbations. Allergy 2018; 73: 50-63.

5.- CHEN J, ZHOU Y, NIE J, WANG Y, ZHANG L, SHI $\mathrm{Q}$, et al. Bacterial lysate for the prevention of chronic rhinosinusitis recurrence in children. J Laryngol Otol 2017; 131: 523-8.

6.- JURKIEWICZ D, ZIELNIK-JURKIEWICZ B. Bacterial lysates in the prevention of respiratory tract infections. Otolaryngol Pol 2018; 72: 1-8.

7.- KEARNEY SC, DZIEKIEWICZ M, FELESZKO W. Immunoregulatory and immunostimulatory responses of bacterial lysates in respiratory infections and asthma. Ann Allergy Asthma Immunol 2015; 114: 364-9.

8.- ESPOSITO S, SOTO-MARTÍNEZ ME, FELESZKO W, JONES MH, SHEN KL, SCHAAD UB. Nonspecific immunomodulators for recurrent respiratory tract infections, wheezing and asthma in children: a systematic review of mechanistic and clinical evidence. Curr Opin Allergy Clin Immunol 2018; 18: 198-209.

9.- DE BENEDETTO F, SEVIERI G. Prevention of respiratory tract infections with bacterial lysate OM-85 bronchomunal in children and adults: a state of the art. Multidiscip Respir Med 2013; 8: 33.

10.- LIU C, HUANG R, YAO R, YANG A. The immunotherapeutic role of bacterial lysates in a mouse model of asthma. Lung 2017; 195: 563-9.

11.- JUST J, BOURGOIN-HECK M, AMAT F. Clinical phenotypes in asthma during childhood. Clin Exp Allergy 2017; 47: 848-55.

12.- SIGURS N, ALJASSIM F, KJELLMAN B, ROBINSON PD, SIGURBERGSSON F, BJARNASON R, et al. Asthma and allergy patterns over 18 years after severe RSV bronchiolitis in the first year of life. Thorax 2010; 65: 1045-52.

13.- JACKSON D, GANGON R, EVANS M, ROBERG K, ANDERSON E, PAPPAS T, et al. Wheezing Rhinovirus illnesses in early life predict asthma development in high-risk children. Am J Respir Crit Care Med 2008; 178: $667-72$.
14.- YIN J, XU B, ZENG X, SHEN K. Broncho-Vaxom in pediatric recurrent respiratory tract infections: A systematic review and meta-analysis. Int Immunopharmacol 2018; 54: 198-209.

15.- CHEN ZG, JI J, LI M, CHEN YF, CHEN FH, CHEN $\mathrm{H}$. Immunoregulants improves the prognosis of infants with wheezing. Nan Fang YiKe Da Xue Xue Bao 2007; 27: 1612-3.

16.- LIAO JY, ZHANG T. Influence of OM-85 BV on hBD-1 and immunoglobulin in children with asthma and recurrent respiratory tract infection. Zhongguo Dang Dai ErKeZaZhi 2014; 16: 508-12.

17.- LU Y, LI Y, XU L, XIA M, CAO L. Bacterial lysate increases the percentage of natural killer $\mathrm{T}$ cells in peripheral blood and alleviates asthma in children. Pharmacology 2015; 95: 139-44.

18.- RAZI CH, HARMANCI K, ABACI A, ÖZDEMIR O, HIZLI S, RENDA R, et al. The immunostimulant OM$85 \mathrm{BV}$ prevents wheezing attacks in preschool children. J Allergy Clin Immunol 2010; 126: 763-9.

19.- JAREONCHARSRI P, BUNNAG C, TUNSURIYAWONG P, ASSANASANE P, VORAPRAYOON S, PINKAEW B. An open-label, prospective study of an oral polyvalent bacterial lysate (Luivac) in the treatment of recurrent respiratory tract infections in Thai patients. Asian Pac J Allergy Immunol 2003; 21: 223-30.

20.- MONIUSZKO T, RUTKOWSKI R, CHYREK-BOROWSKA S. Effect of Ribomunyl therapy on production of selected cytokines by mononuclear peripheral blood cells in nonatopic bronchial asthma. Pneumono Alergol Pol 1995; 63 Suppl 2: 71-5.

21.- SABBAH A. An immunoregulator of asthma: Ribomunyl. Allerg Immunol (Paris) 1986; 18: 41-3.

22.- EMERYK A, BARTKOWIAK-EMERYK M, RAUS Z, BRAIDO F, FERLAZZO G, MELIOLI G. Mechanical bacterial lysate administration prevents exacerbation in allergic asthmatic children-The EOLIA study. Pediatr Allergy Immunol 2018; 29: 394-401.

23.- LAU S, GERHOLD K, ZIMMERMANN K, OCKELOEN CW, ROSSBERG S, WAGNER P, et al. Oral application of bacterial lysate in infancy decreases the risk of atopic dermatitis in children with 1 atopic parent in a randomized, placebo-controlled trial. Allergy Clin Immunol 2012; 129: 1040-7.

Correspondencia a:

Dr. Daniel Zenteno Araos

Depto. de Pediatría, Universidad de Concepción.

Avenida Alemana 98 Pedro de Valdivia. Concepción,

Chile.

Email: danielzenteno@gmail.com 